\title{
Bayesian Stochastic Frontier Analysis Using WinBUGS
}

\author{
J.E. Griffin* and M.F.J. Steel \\ Department of Statistics, University of Warwick, Coventry, CV4 7AL, U.K.
}

\begin{abstract}
Markov chain Monte Carlo (MCMC) methods have become a ubiquitous tool in Bayesian analysis. This paper implements MCMC methods for Bayesian analysis of stochastic frontier models using the WinBUGS package, a freely available software. General code for cross-sectional and panel data are presented and various ways of summarizing posterior inference are discussed. Several examples illustrate that analyses with models of genuine practical interest can be performed straightforwardly and model changes are easily implemented. Although WinBUGS may not be that efficient for more complicated models, it does make Bayesian inference with stochastic frontier models easily accessible for applied researchers and its generic structure allows for a lot of flexibility in model specification.
\end{abstract}

Keywords: Efficiency, Markov chain Monte Carlo, Model comparison, Regularity, Software

\section{Introduction}

The use of stochastic frontiers in the analysis of productivity and firm efficiency has become widespread since the seminal papers by Meeusen and van den Broeck (1977) and Aigner, Lovell and Schmidt (1977). More recently, a large amount of interest has been devoted to the use of Bayesian methods for making inference in stochastic frontier models. The latter was introduced by van den Broeck et al. (1994), who commented on its particular advantages in this context: exact (small-sample) inference on efficiencies, easy incorporation of prior ideas and restrictions such as regularity conditions and formal treatment of parameter and model uncertainty. Bayesian methods are now commonplace in this literature, as evidenced by Kim and Schmidt (2000) and recent applications by e.g. Kurkalova and Carriquiry (2002) and Ennsfellner, Lewis and Anderson (2004). In addition, five of the 12 papers in a recent special issue of the Journal of Econometrics on "Current developments in productivity and efficiency measurement" (Dorfman and Koop, 2005) adopt a Bayesian approach. The complexity of stochastic frontier models

\footnotetext{
${ }^{*}$ Corresponding author: Jim Griffin, Department of Statistics, University of Warwick, Coventry, CV4 7AL, U.K. Tel.: +441227-82 3865; Fax: +44-24-7652 4532; Email: J.E.Griffin@warwick.ac.uk.
} 
makes numerical integration methods inevitable. The most appropriate method in this context is clearly Markov chain Monte Carlo (MCMC), as introduced by Koop, Steel and Osiewalski (1995) and used in virtually all recent Bayesian papers in this literature, see e.g. Kurkalova and Carriquiry (2002), Tsionas (2002), Huang (2004) and Kumbhakar and Tsionas (2005).

A problem that occurs, especially for applied users that have not yet implemented Bayesian methods in this field, is the availability of reliable and user-friendly software. To our knowledge, there is only one publicly available software, which is described in Arickx et al. (1997). However, the latter is based on importance sampling, rather than MCMC, and lacks flexibility in model specification (it basically only implements the simple cross-sectional stochastic frontier model with a restricted choice of efficiency distributions and priors). ${ }^{1}$

Thus, this paper describes the use of a freely available software for the analysis of complex statistical models using MCMC techniques, called WinBUGS, in the context of stochastic frontiers. It turns out that WinBUGS can become quite a powerful and flexible tool for Bayesian stochastic frontier analysis, and only requires a relatively small investment on the part of the user. Once the (applied) user understands the logic of model building with WinBUGS, Bayesian analysis is conducted quite easily and many built-in features can be accessed to produce an in-depth and interactive analysis of these models. In addition, execution is reasonably fast ${ }^{2}$, even of complicated models with large amounts of data and model extensions can easily be accommodated in a modular fashion. The modeller can really concentrate on building and refining an appropriate model ${ }^{3}$, without having to invest large amounts of time in coding up the MCMC analysis and the associated processing of the results. Despite the relative ease of use, we do wish to reiterate the health warning that comes with WinBUGS: "The programs are reasonably easy to use and come with a wide range of examples. There is, however, a need for caution. A knowledge of Bayesian statistics is assumed, including recognition of the potential importance of prior distributions, and MCMC is inherently less robust than analytic statistical methods. There is no in-built protection against misuse."

WinBUGS is a generic tool which can be used in a wide variety of situations. Thus, it can not be expected to be quite as efficient as tailor-made MCMC implementations designed specifically for certain models. However, given the continuing introduction of new stochastic frontier models, the availability of a flexible computational environment that can easily be adapted to new settings is of great practical value, especially in an exploratory stage, where we may not want to invest heavily in coding up a particular model in the most efficient manner.

\footnotetext{
${ }^{1}$ Other Bayesian software can perhaps be adapted to deal with stochastic frontier models. For example, Koop (1999) suggests that BACC (Bayesian Analysis, Computation and Communication) software might be extended to cover stochastic frontier models, but does not provide any details on such an implementation.

${ }^{2}$ The basic model takes about 100 seconds for 100,000 draws on a Xeon $3.06 \mathrm{GHz}$ PC with the cross-sectional electricity data used in Section 3, whereas one of the most time-consuming models, the truncated normal with the inefficiencies depending on covariates requires avout 7.5 hours for 100,000 draws using the large hospital panel data set in Section 4 . In the second case, these draws need to be thinned (we use every 100th draw), making a large run relatively time-consuming with this standard WinBUGS code. A specifically tailored add-on to WinBUGS could improve the efficiency dramatically for these more challenging models.

${ }^{3}$ This can include models which have not previously appeared in the literature.
} 
We illustrate the use and flexibility of WinBUGS for Bayesian stochastic frontier modelling of the cross-sectional data on electric utility companies used and listed in Greene (1990) and of the panel data on hospitals used in Koop, Osiewalski and Steel (1997).

The WinBUGS software (together with a user manual) can be downloaded (the current fee is zero) from the website

http://www.mrc-bsu.cam.ac.uk/bugs/winbugs/contents.shtml.

We have used Version 1.4 in this paper. All WinBUGS code used in this paper, as well as the data on the electricity firms and hospitals is freely available at

http://www.warwick.ac.uk/go/msteel/steel_homepage/software/.

\section{Stochastic Frontier Model}

The basic model relates producers's costs (or outputs) to a minimum cost (or maximum output) frontier. If a panel of costs has been observed, a simple model regresses the logarithm of cost, $y_{i t}$, associated with firm $i$ observed at time $t$ and producing a certain quantity $Q_{i t}$, on a set of regressors in $x_{i t}$, which will be functions of the logarithm of input prices and $Q_{i t}(i=1, \ldots, N, t=1, \ldots, T)$ :

$$
y_{i t} \stackrel{i n d}{\sim} \mathrm{N}\left(\alpha+x_{i t}^{\prime} \beta+u_{i t}, \sigma^{2}\right)
$$

where $\mathrm{N}\left(\mu, \sigma^{2}\right)$ denotes a normal distribution with mean $\mu$ and variance $\sigma^{2}$. Inefficiencies $u_{i t}$ model the difference between best-practice and actual cost, and these are assumed to have a one-sided distribution, such as the exponential (as e.g. in Meeusen and van den Broeck, 1977). Often we will exploit the panel context by assuming that inefficiencies remain constant over time, i.e. $u_{i t}=u_{i}, t=1, \ldots, T$, leading to $^{4}$

$$
u_{i} \stackrel{i . i . d .}{\sim} \operatorname{Exp}(\lambda)
$$

which denotes an exponential distribution with mean $1 / \lambda$. The parameters introduced in this model are assigned priors, for example a multivariate normal:

$$
\beta \sim \mathrm{N}(0, \Sigma)
$$

possibly truncated to reflect regularity conditions (see Subsection 3.3),

$$
\sigma^{-2} \sim \mathrm{Ga}\left(a_{0}, a_{1}\right)
$$

a gamma distribution with shape parameter $a_{0}$ and mean $a_{0} / a_{1}$, and

$$
\lambda \sim \operatorname{Exp}\left(-\log r^{\star}\right),
$$

where the latter prior implies that prior median efficiency is equal to $r^{\star}$. Minor changes are required for production frontiers. ${ }^{5}$ Firm-specific efficiencies are introduced as functions of the inefficiency terms;

\footnotetext{
${ }^{4}$ For large $T$ that assumption might be weakened, as we will discuss in Subsection 4.2.

${ }^{5}$ If we wish to model production frontiers, $y_{i t}$ will be the output produced with a certain quantity of inputs, which will determine the regressors in $x_{i t}$ and the inefficiency term $u_{i t}$ will appear with a negative sign in the mean of $y_{i t}$.
} 
in particular, the efficiency of firm $i$ is defined as $r_{i}=\exp \left(-u_{i}\right)$. These are clearly key quantities of interest in practice.

In WinBUGS, models are expressed in code through the distributions of the observations and parameters together with their independence structure. A stochastic cost frontier model is formulated in Display 1 for a potentially unbalanced panel of observations with time-invariant inefficiencies. Firstly, the distribution of the log cost in equation (1) is coded. The vector $y$ holds the $K$ observed log costs, and the matrix data is a $K \times(p+2)$ matrix. Each row holds the observed values for each firm of the $\mathrm{p}$ regressors, the $\mathrm{p}+1$-th column holds an index for each firm running from 1 to $\mathrm{N}$ and the $\mathrm{p}+2$-th column holds the time of the observation, which has a maximum value of $\mathrm{T}$ (the latter variable is used in Section 4, where we have a time trend in the frontier and allow for efficiencies to vary over time). The distribution of $y_{i t}$ is encoded using the command dnorm for the stochastic node $\mathrm{y}[\mathrm{k}]$ which has two arguments representing the mean and the precision (the inverse of the variance). WinBUGS restricts the parameters of the command to be variables and so the mean must be defined as a logical node mu [ $\mathrm{k}$ ] which can be formed in a standard way. The expression inprod (beta [ ] data $[k, 1: p]$ represents $x_{i t}^{\prime} \beta$.

The inefficiency $u_{i}$ is specified to have an exponential distribution with mean $1 / \lambda$ using the command dexp (lambda). A useful feature of WinBUGS is the use of logical nodes to define interesting functions of the parameters in the model. The $i$-th firm's efficiency is represented by eff [i]. Finally, the prior distributions of each unknown parameter is specified as above.

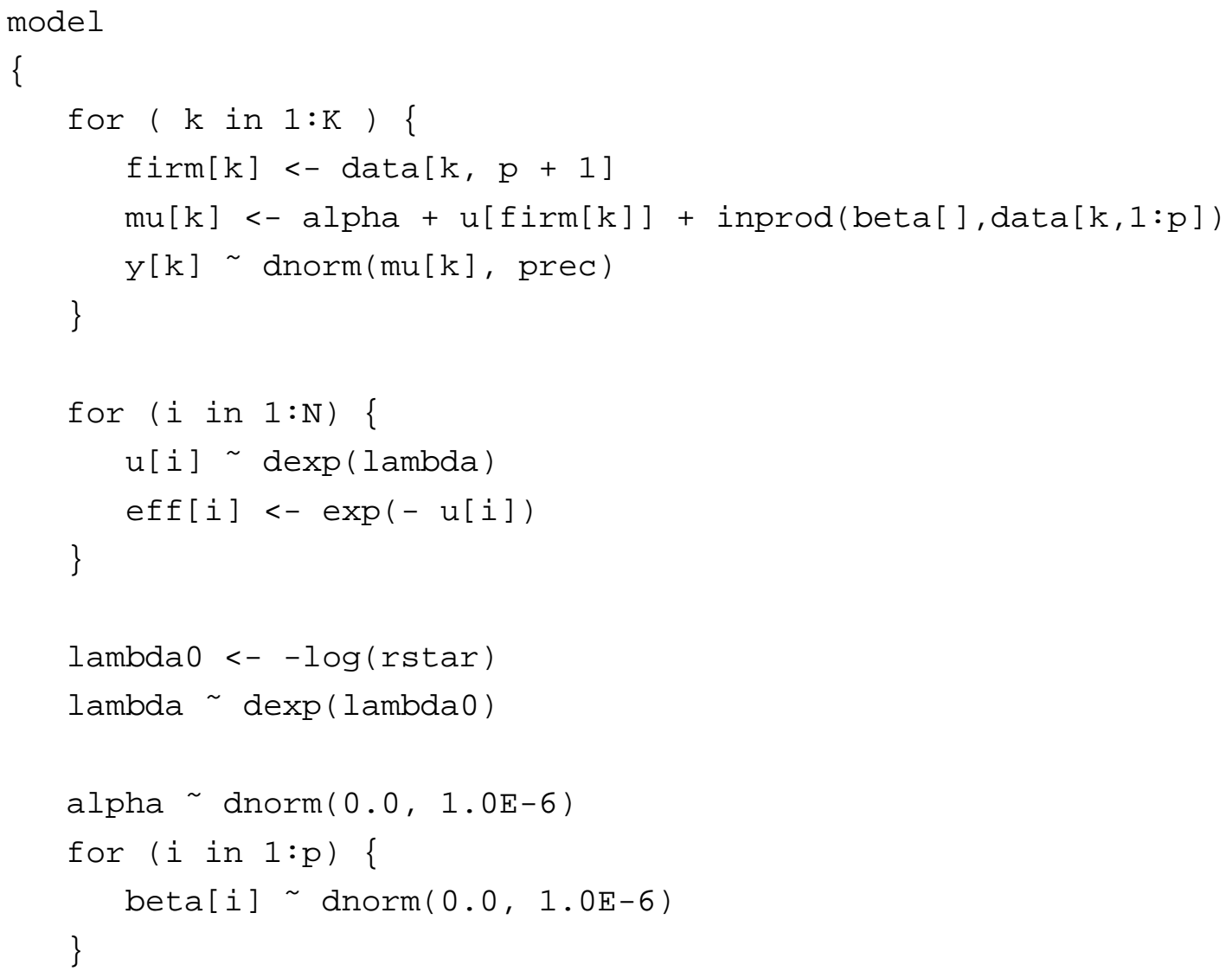




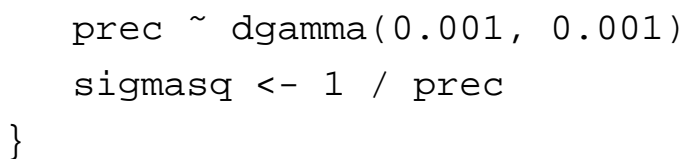

Display 1: WinBUGS model specification code for the basic cost frontier with panel data

It is very easy to change some of the model assumptions above. For example, we may want to use a different distribution for the inefficiencies, such as the half-normal used in Aigner, Lovell and Schmidt (1977) or the truncated normal of Stevenson $(1980)^{6}$. For the half-normal

$$
u_{i} \sim \mathrm{N}^{+}\left(0, \lambda^{-1}\right)
$$

we simply replace the distribution of $u[i]$ by

$u[i] \sim d j l . d n o r m . t r u n c(0,1$ ambda, 0, 1000),

while the truncated normal distribution

$$
u_{i} \sim \mathrm{N}^{+}\left(\zeta, \lambda^{-1}\right)
$$

also allows the mean of the underlying normal distribution to be estimated and is implemented by

u[i] djl.dnorm.trunc(zeta, lambda, 0,1000)

A general gamma $\mathrm{Ga}(\phi, \lambda)$ distribution as in Greene (1990) would correspond to

u[i] dgamma (phi, lambda).

Appropriate prior specifications for the parameters need to be included. Various suggestions for prior choices have been made in the literature (e.g. in Tsionas, 2000 and Griffin and Steel, 2004b).

Once the model code has been loaded, the data must be specified in a special format, which is taken from the statistical package, S-plus. More details are available from the WinBUGS manual. Code for converting data from some other popular packages can be found on the page

http://www.mrc-bsu.cam.ac.uk/bugs/weblinks/webresource.shtml

Finally, initial values for the variables being estimated need to be specified. The speed of convergence of the chain is affected by these values. Values with larger posterior density will generally lead to faster convergence. However, in our experience convergence of chains is relatively fast from most plausible choices. Once the model, data and initial values have been entered, WinBUGS creates compiled code to perform an MCMC algorithm for sampling from the posterior distribution. There are several sampling options including multiple chains to aid convergence diagnosis and thinning of the chain to reduce dependence between successive simulated values.

\footnotetext{
${ }^{6}$ The truncated normal and half-normal need a "shared component" which can be downloaded from the WinBUGS development site, currently at http://www.winbugs-development.org.uk/shared.html.
} 
The following sections illustrate the power of WinBUGS to produce useful summary statistics and graphical representations of the posterior distribution with several example datasets. We also show how changes to the model specification can be implemented quite easily, and how to deal with economic regularity conditions and model uncertainty. In addition, we discuss how to implement dependence of the efficiencies on covariates that can vary over firms and time.

\section{An Old Chestnut: The Electricity Data}

The first example analyses $N=123$ cross-sectional data from the U.S. electric utility industry in 1970 . The data was originally analysed by Christensen and Greene (1976) and subsequently by Greene (1990). Following that analysis, we specify the frontier for $\log \left(\operatorname{Cost} / P_{f}\right)$ as

$$
\alpha+\beta_{1} \log Q+\beta_{2} \log \left(P_{l} / P_{f}\right)+\beta_{3} \log \left(P_{k} / P_{f}\right)+\beta_{4} \log ^{2} Q
$$

where Output $(Q)$ is produced with three factors: labour, capital, and fuel and the respective factor prices are $P_{l}, P_{k}$ and $P_{f}$.

\subsection{The standard exponential model}

Here we use the model in Section 2, where now we have $T=1$, with exponentially distributed inefficiencies. The chain was run with a burn-in of 20000 iterations with 200000 retained draws and a thinning to every 5th draw. WinBUGS has a number of tools for reporting the posterior distribution. A simple summary (Table 1) can be generated showing posterior mean, median and standard deviation with a $95 \%$ posterior credible interval. Parameter names are related in an obvious way to the model in Section 2. A fuller picture of the posterior distribution can be provided using the density option in the Sample Monitor Tool which draws a kernel density estimate of the posterior distribution of any chosen parameter, as in Figure 1.

$\begin{array}{lllllllll}\text { node } & \text { mean } & \text { sd } & \text { MC error } & \mathbf{2 . 5 \%} & \text { median } & \mathbf{9 7 . 5 \%} & \text { start } & \text { sample } \\ \text { alpha } & -7.47 & 0.3407 & 0.001049 & -8.133 & -7.472 & -6.793 & 20001 & 200000 \\ \text { beta[1] } & 0.4252 & 0.04301 & 1.544 \mathrm{E}-4 & 0.34 & 0.4255 & 0.5088 & 20001 & 200000 \\ \text { beta[2] } & 0.2501 & 0.06495 & 1.513 \mathrm{E}-4 & 0.1226 & 0.2499 & 0.3776 & 20001 & 200000 \\ \text { beta[3] } & 0.0473 & 0.06193 & 1.823 \mathrm{E}-4 & -0.07302 & 0.04679 & 0.17 & 20001 & 200000 \\ \text { beta[4] } & 0.02962 & 0.002843 & 8.653 \mathrm{E}-6 & 0.02407 & 0.02961 & 0.03524 & 20001 & 200000 \\ \text { lambda } & 12.23 & 5.207 & 0.03901 & 7.072 & 10.79 & 26.75 & 20001 & 200000 \\ \text { sigmasq } & 0.01332 & 0.003836 & 2.381 \mathrm{E}-5 & 0.007233 & 0.01281 & 0.02188 & 20001 & 200000\end{array}$

Table 1: WinBUGS output for the electricity data: posterior statistics

Often, the quantities of primary interest in stochastic frontier analysis are the efficiencies. Firmspecific efficiencies are immediately generated by the sampler for each firm and their full posterior distributions are readily available, and can be plotted in the same way as in Figure 1. There are various other 

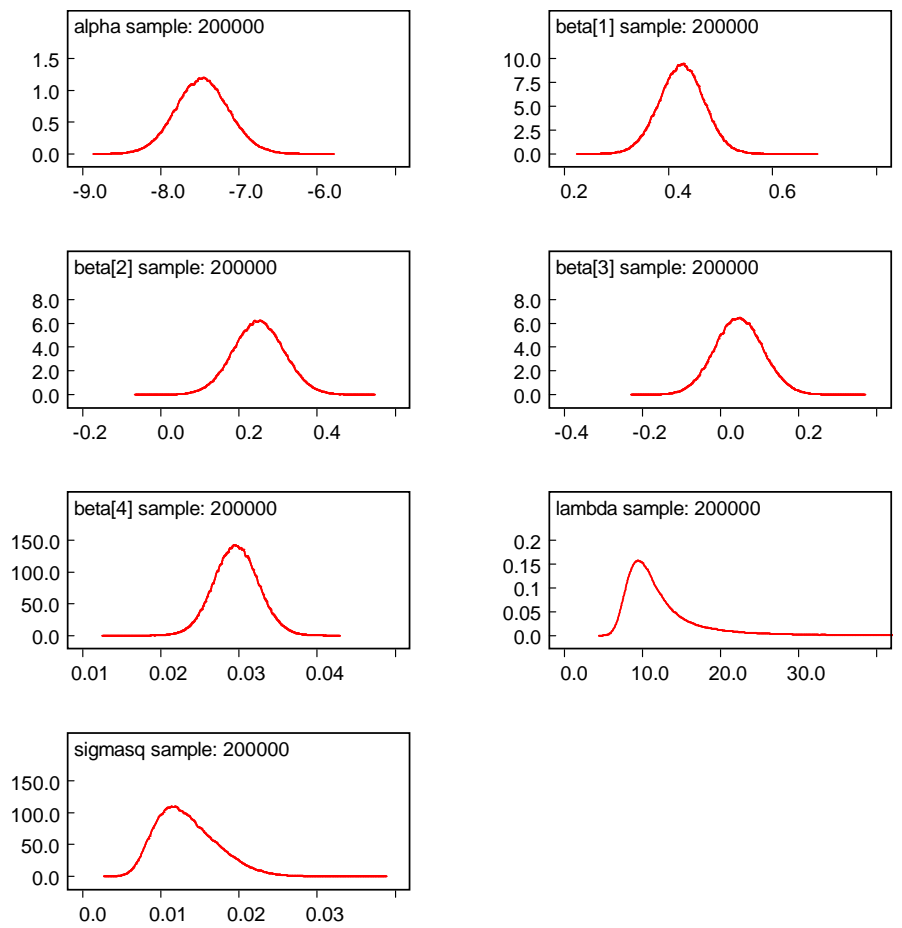

Figure 1: WinBUGS output for the electricity data: posterior densities for parameters

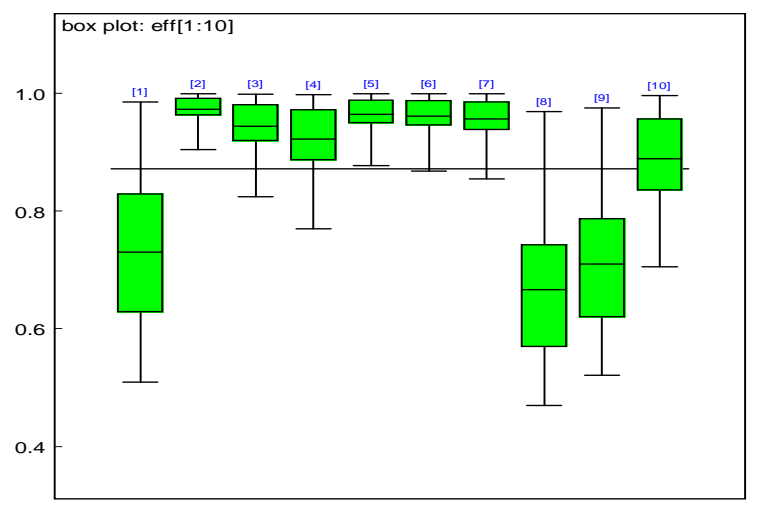

Figure 2: WinBUGS output for the electricity data: boxplot of posterior efficiency distributions for the first ten firms in the sample

options for displaying the posterior distribution. For example the Compare... menu item brings up the Comparison Tool that draws a boxplot (Figure 2) or caterpillar plot of the sampled efficiencies for some chosen firms (in this case, the first ten firms in the sample). A practically interesting function of the firm-specific efficiency measurements is given by their ranks. WinBUGS can automatically compute a sample from their posterior distribution using the Rank... option from the Inference menu. Table 2 shows a summary of the posterior distribution for the first seven electricity producers in the sample (high rank corresponds to high efficiencies). The posterior distribution clearly demonstrates a large spread of the rankings. 


$\begin{array}{rlll}\text { node } & \mathbf{2 . 5 \%} & \text { median } & \mathbf{9 7 . 5 \%} \\ \text { eff[1] } & 1 & 4 & 90 \\ \text { eff[2] } & 34 & 99 & 123 \\ \text { eff[3] } & 13 & 74 & 121 \\ \text { eff[4] } & 9 & 58 & 120 \\ \text { eff[5] } & 25 & 91 & 122 \\ \text { eff[6] } & 23 & 89 & 122 \\ \text { eff[7] } & 21 & 84 & 122\end{array}$

Table 2: WinBUGS output for the electricity data: rank statistics for the first seven firms in the sample

A simple check of the mixing of the posterior distribution arises from a graphical summary of the values taken by the chain. For example, a trace plot of all drawn values is available through the history button or through the trace button for the previous batch of, say, 1000 drawings.
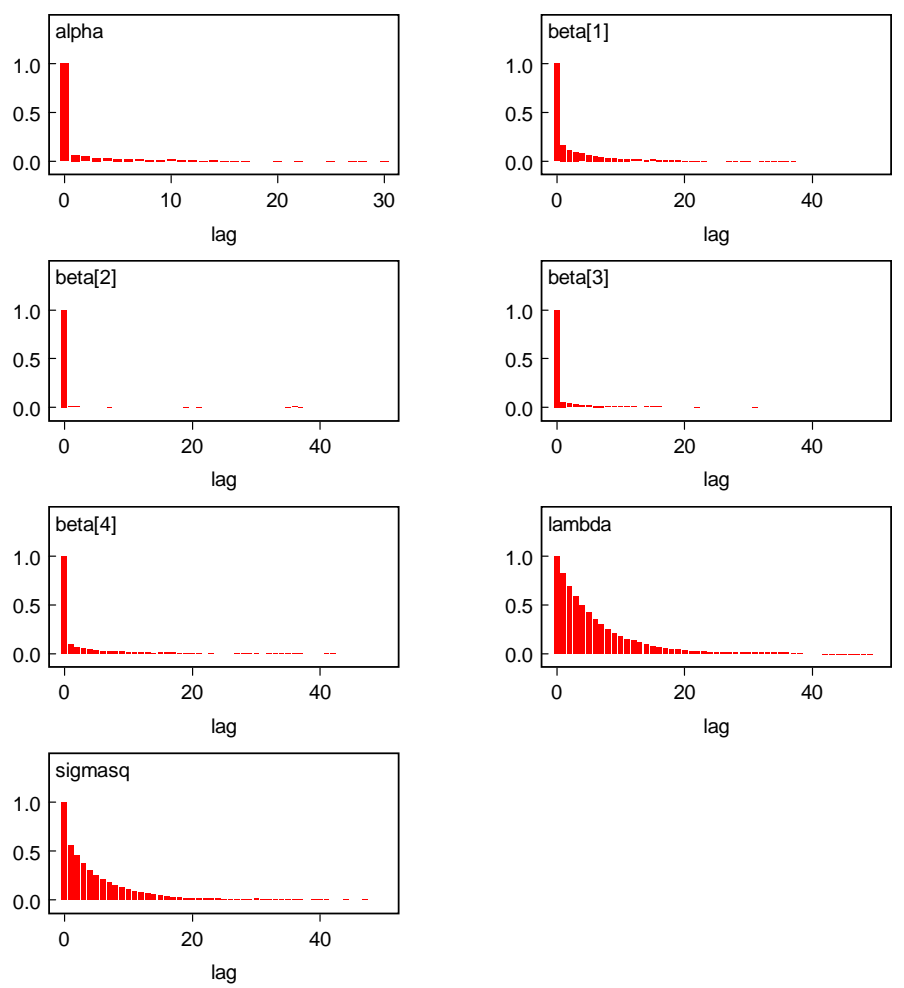

Figure 3: WinBUGS output for the electricity data: autocorrelation functions of the chain

The autocorrelation function for the chain of each parameter (as shown in Figure 3) can also indicate dimensions of the posterior distribution that are mixing slowly. Slow mixing is often associated with high posterior correlations between parameters. The plots indicate that all parameters are mixing well with autocorrelation vanishing before 20 lags in each case. The correlation tool (the correlation 
option in the inference menu) can produce scatterplots of every parameter against every other parameter to indicate correlation or a correlation coefficient can be estimated from the current output.

Graphical representations of the posterior distribution can indicate problems with the performance of the MCMC algorithm. More sophisticated methods for convergence detection are implemented in the Convergence Diagnostic and Output Analysis (CODA) software which is available for the statistical packages S-plus and R. WinBUGS produces output that is formatted for direct use with these programs and allows the behaviour of the chain to be investigated using some popular statistical tests.

$$
\begin{aligned}
& \text { Dbar }=\text { post.mean of }-2 \log L ; \text { Dhat }=-2 \log L \text { at post.mean of stochastic nodes } \\
& \text { Dbar Dhat pD DIC } \\
& \text { y } \quad-187.486 \quad-233.447 \quad 45.961 \quad-141.525 \\
& \text { total }-187.486-233.447 \quad 45.961 \quad-141.525
\end{aligned}
$$

Table 3: WinBUGS output for the electricity data: DIC with normal errors

WinBUGS automatically implements the DIC (Spiegelhalter et al., 2002) model comparison criterion. This is a portable information criterion quantity that trades off goodness-of-fit against a model complexity penalty. In hierarchical models, deciding the model complexity may be difficult and the method estimates the "effective number of parameter", denoted here by $p_{D} . \bar{D}$ is the posterior mean of the deviance $(-2 \times \log$ likelihood $)$ and $\hat{D}$ is a plug-in estimate of the latter based on the posterior mean of the parameters. The DIC is computed as DIC $=\bar{D}+p_{D}=\hat{D}+2 p_{D} .^{7}$ Lower values of the criterion indicate better fitting models. Table 3 records the values computed, in the format given by WinBUGS. For our purposes here, we will focus only on the DIC value. The method was designed to be easy to implement using a sample from the posterior distribution and the interested reader is directed to Spiegelhalter et al. (2002) for a lively discussion of its merits and its relation to the more usual Bayes factor ${ }^{8}$.

\subsection{Alternative distributional assumptions}

Once this model has been fitted successfully, we may want to consider further modelling options. As already indicated in Section 2, there are alternative choices of the inefficiency distribution. Another popular choice is the half-normal, which can be implemented in WinBUGS by

$u[i] \sim$ djl.dnorm.trunc $(0$, lambda, 0,1000$)$.

The prior distribution

lambda dgamma $(1,1 / 37.5)$

${ }^{7}$ Thus, $p_{D}$ is computed as $\bar{D}-\hat{D}$.

${ }^{8}$ Model comparison using DIC can, in some cases, lead to different conclusions that those obtained through Bayes factors. The calculation of Bayes factors from MCMC output is non-trivial and is not easily implemented in WinBUGS. A variety of methods is available for the computation of Bayes factors, and these could be implemented in problem-specific coding in a standard computer language, or in an add-on that could be written for WinBUGS. 
leads to a prior median efficiency of approximately 0.875 with a reasonable spread (see van den Broeck et al., 1994, taking $\nu_{0}=2$ in their (29) and $a=0$ corresponding to the half-normal). Yet another possible inefficiency distribution is the gamma distribution, implemented by

u[i] dgamma (phi, lambda).

A suitable prior distribution, which extends the informative prior for an exponential inefficiency distribution, is discussed in Griffin and Steel (2004b) in a more general setting. They define

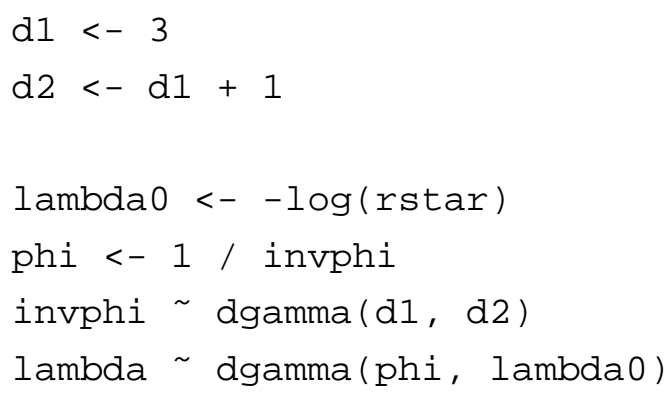

The prior on phi has mode at one (corresponding to the exponential), so this centres the gamma distribution over the exponential distribution and the parameter $\mathrm{d} 1$ controls the variability of phi (a value of 3 is their suggested setting).

\begin{tabular}{|c|c|c|c|}
\hline inefficiency distribution & exponential & half-normal & gamma \\
\hline$\alpha$ & $-7.5(-8.1,-6.8)$ & $-7.4(-8.0,-6.7)$ & $-7.5(-8.1,-6.8)$ \\
$\beta_{1}$ & $0.43(0.34,0.51)$ & $0.41(0.33,0.48)$ & $0.42(0.33,0.50)$ \\
$\beta_{2}$ & $0.25(0.12,0.38)$ & $0.24(0.11,0.37)$ & $0.25(0.12,0.38)$ \\
$\beta_{3}$ & $0.05(-0.07,0.17)$ & $0.06(-0.06,0.19)$ & $0.05(-0.07,0.18)$ \\
$\beta_{4}$ & $0.030(0.024,0.035)$ & $0.031(0.025,0.036)$ & $0.030(0.024,0.036)$ \\
$\phi$ & & & $1.65(0.58,6.15)$ \\
$\lambda$ & $10.8(7.1,26.8)$ & $44.5(20.8,140.6)$ & $14.5(6.9,52.1)$ \\
$\sigma^{2}$ & $0.013(0.007,0.022)$ & $0.013(0.006,0.021)$ & $0.013(0.006,0.013)$ \\
\hline
\end{tabular}

Table 4: Parameter results (posterior medians and 95\% credible intervals) for various efficiency distributions with normal errors for the electricity data

Table 4 contrasts some results on the parameters for exponential, half-normal ${ }^{9}$ and gamma assumptions. Differences on common parameters are fairly small ${ }^{10}$, and the credible interval for $\phi$, the shape parameter of the gamma, includes 1 , which corresponds to the exponential model.

In addition, a heavier tailed error distribution could be considered for the measurement error. Simply specifying

$y[k] \sim d t(m u[k]$, prec, degfree)

\footnotetext{
${ }^{9}$ The more general truncated normal will be used in the context of the hospital panel data in the next application.

${ }^{10}$ Note that $\lambda$ does not share a common interpretation across models.
} 
changes the form of the error distribution in (1) to a $t$-distribution with degfree degrees of freedom. The prior distribution for the degrees of freedom was chosen to be exponential with mean and standard deviation equal to 3 :

degfree $\operatorname{dexp}(0.333)$,

which puts a considerable amount of prior mass on distributions with much heavier tails than the normal distribution.

\begin{tabular}{|c|c|c|c|}
\hline inefficiency distribution & exponential & half-normal & gamma \\
\hline$\alpha$ & $-7.8(-8.4,-7.1)$ & $-7.7(-8.4,-7.0)$ & $-7.8(-8.4,-7.1)$ \\
$\beta_{1}$ & $0.45(0.36,0.52)$ & $0.44(0.35,0.52)$ & $0.44(0.35,0.52)$ \\
$\beta_{2}$ & $0.29(0.16,0.42)$ & $0.28(0.16,0.41)$ & $0.29(0.16,0.42)$ \\
$\beta_{3}$ & $0.04(-0.07,0.16)$ & $0.05(-0.06,0.17)$ & $0.04(-0.07,0.16)$ \\
$\beta_{4}$ & $0.028(0.023,0.034)$ & $0.029(0.023,0.034)$ & $0.023(0.028,0.034)$ \\
$\nu$ & $4.4(2.0,11.8)$ & $3.5(1.6,10.3)$ & $4.0(1.7,11.1)$ \\
$\phi$ & & & $1.9(0.7,6.9)$ \\
$\lambda$ & $11.3(7.1,29.4)$ & $43.8(20.8,141.1)$ & $16.0(7.5,56.1)$ \\
$\sigma^{2}$ & $0.008(0.003,0.016)$ & $0.006(0.001,0.014)$ & $0.002(0.0004,0.0073)$ \\
\hline
\end{tabular}

Table 5: Parameter results (posterior medians and 95\% credible intervals) for the two alternative efficiency distributions with $t$ errors for the electricity data

Table 5 records some results and illustrates that the prior assumption about the degrees of freedom, indicated by $\nu$, is quite important since the data provide little information about its value. We can use the DIC criterion to compare the different models. Table 6 compares the DIC scores for the possible

\begin{tabular}{|l|l|cccc|}
\hline error distribution & inefficiency distribution & $\bar{D}$ & $\hat{D}$ & $p_{D}$ & $D I C$ \\
\hline normal & exponential & -187.5 & -233.4 & 46.0 & -141.5 \\
& half-normal & -189.3 & -239.5 & 50.2 & -139.1 \\
& gamma & -187.7 & -234.0 & 46.4 & -141.3 \\
\hline$t$ & exponential & -190.7 & -238.8 & 48.1 & -142.6 \\
& half-normal & -209.7 & -263.3 & 53.6 & -156.1 \\
& gamma & -198.7 & -247.7 & 49.0 & -149.7 \\
\hline
\end{tabular}

Table 6: Comparison of models with different distributional assumptions using the DIC criterion

combinations of error distribution and inefficiency distribution. Smaller values of the DIC suggest better models and so the Student- $t$ errors tend to fit the data better than the normal measurement errors. Overall, the results favour the half-normal distribution with $t$-distributed errors. The posterior distribution of the mean of the predictive (i.e. out-of-sample) efficiency is a useful measure for comparing our inference about the parameters of the inefficiency distributions $\lambda$ and $\phi$ (presented in Table 5). If $t$-distributed errors 


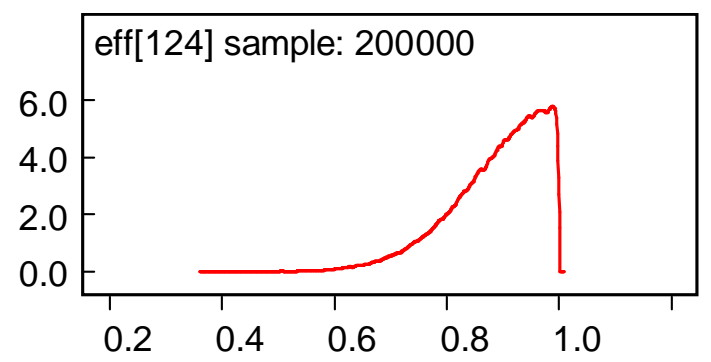

Figure 4: WinBUGS output for the electricity data: kernel density estimate of the posterior predictive efficiency distribution for the Student- $t$ model with half-normal efficiencies

are assumed, the posterior mean of predictive efficiency has a $95 \%$ credibility interval of $(0.88,0.97)$ for the exponential distribution, $(0.80,0.96)$ for the gamma and $(0.85,0.94)$ for the half-normal distribution. In other words, for this data, the half-normal and gamma distributions are associated with slightly lower estimates of efficiency than the exponential distribution.

Finally, we estimate the posterior predictive distribution of efficiency for our preferred model with $t$-distributed measurement errors and a half-normal inefficiency distribution. This corresponds to the efficiency of an unobserved firm in this sector. An extra inefficiency node $(u[N+1])$ and efficiency node (eff $[\mathrm{N}+1]$ ) are added to the model by simply changing the range of the for loop, i.e. defining

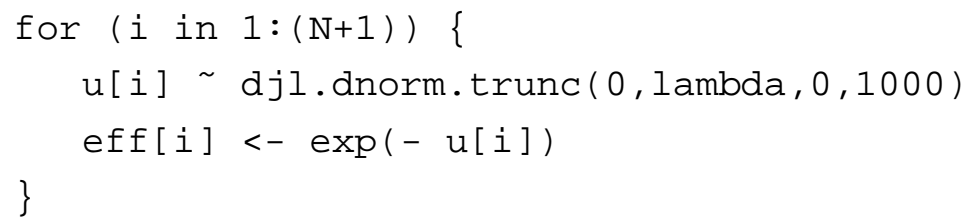

A kernel density estimate of the predictive efficiency distribution, i.e. of eff $[\mathrm{N}+1]$, is shown in Figure 4. Posterior predictive median efficiency is 0.91 and the $95 \%$ credible interval is $(0.69,0.996)$.

\subsection{Imposing regularity conditions}

The fitted frontier should obey certain economic constraints (see e.g. Kumbhakar and Lovell 2000). For example, a cost frontier should imply positive elasticities of cost with respect to output and prices. In other word, we need to check

$$
\frac{\mathrm{d} \log C}{\mathrm{~d} \log X_{i}}>0
$$

If a Cobb-Douglas frontier is fitted the condition reduces to positive coefficients in the frontier. This change can be easily implemented in the prior distribution of $\beta_{i}$ by replacing the normal prior

beta[i] $\operatorname{dnorm}(0.0,1.0 \mathrm{E}-06)$

with its truncated counterpart

beta[i] djl.dnorm.trunc $(0.0,1.0 \mathrm{E}-0.6,0,1000)$. 
However, a more complicated frontier such as a translog will lead to more complicated expressions for $\mathrm{d} \log C / \mathrm{d} \log X_{i}$. In this example, a quadratic output term is included in the frontier and the elasticity of cost with respect to output has the form

$$
\frac{\mathrm{d} \log C}{\mathrm{~d} \log Q}=\beta_{1}+2 \beta_{4} \log Q .
$$

Ideally, we would want this relationship to be true for all values of $\log Q$. However, since this is only a local approximation to the frontier, it is usual to check the condition for a plausible set of values of $\log Q$. A pragmatic approach restricts attention to the value of the elasticity for the observed output. This approach will be used in this case. Imposing these properties in alternative sets of values as in Terrell (1996) can easily be implemented. The restrictions are imposed on $p(y \mid X, \beta)$ leading to a non-standard likelihood function, which is implemented using the "ones-trick" described in the WinBUGS manual. This introduces a new variable check, which will be zero for all cases where regularity conditions are violated and one elsewhere. The data is now a set of ones assumed to be the result of Bernoulli sampling with probabilities proportional to the likelihood values of those observations for which regularity holds and zero for which it is violated. As soon as a violation occurs for one of the observations, the likelihood value associated with that draw will, thus, be zero. $C$ is a constant such that all values in prob are smaller than one. In particular, we replace (note that the definition of $m u[k]$ now differs from that in Display 1 due to the squared log output term in (3))

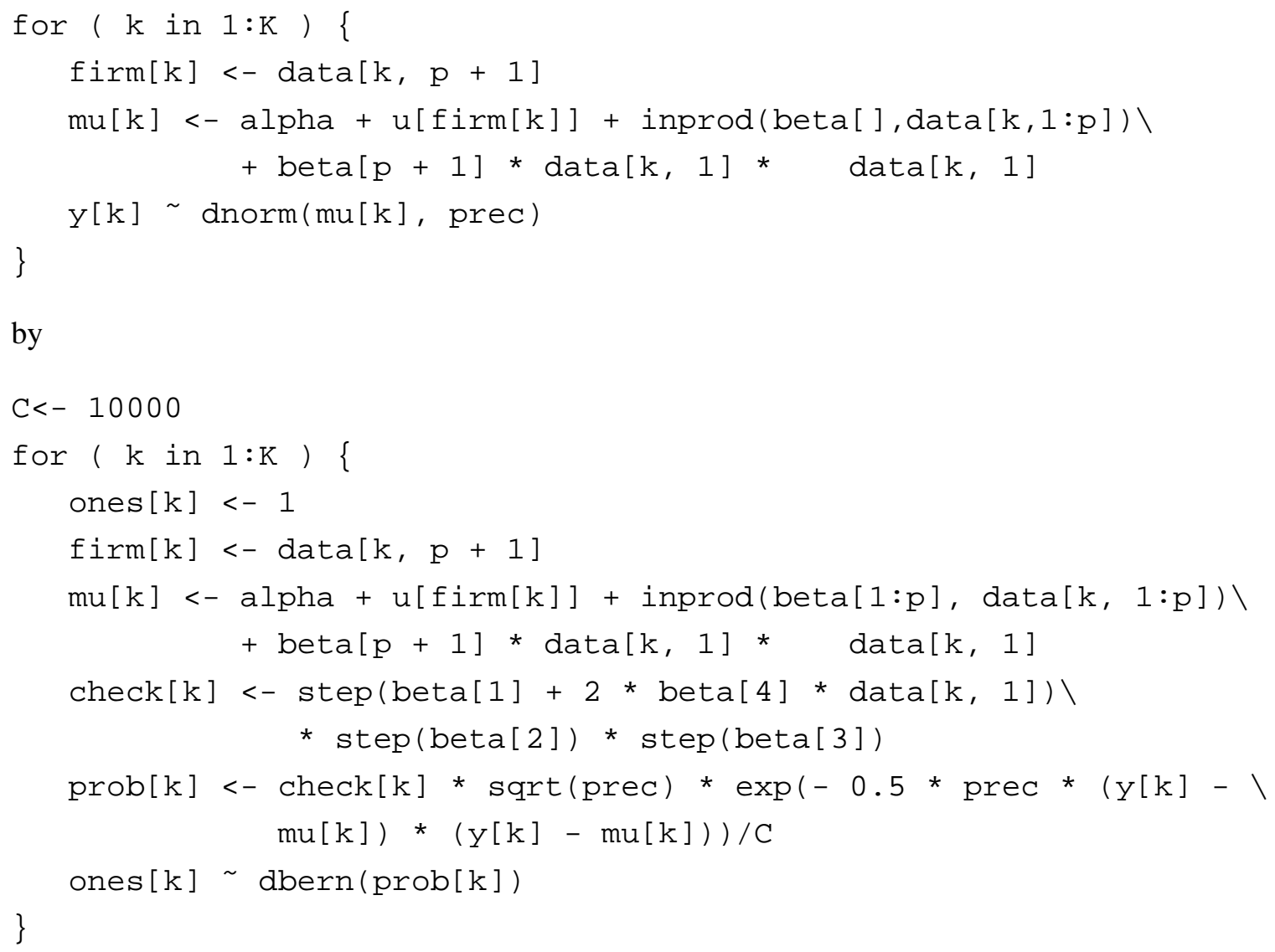




\begin{tabular}{|c|c|c|}
\hline inefficiency distribution & exponential & half-normal \\
\hline$\alpha$ & $-7.7(-8.2,-7.0)$ & $-7.7(-8.3,-7.0)$ \\
$\beta_{1}$ & $0.44(0.35,0.52)$ & $0.44(0.35,0.52)$ \\
$\beta_{2}$ & $0.27(0.16,0.37)$ & $0.27(0.15,0.39)$ \\
$\beta_{3}$ & $0.06(0.00,0.16)$ & $0.06(0.00,0.17)$ \\
$\beta_{4}$ & $0.028(0.023,0.034)$ & $0.028(0.023,0.034)$ \\
$\nu$ & $4.4(2.1,11.8)$ & $3.6(1.6,10.4)$ \\
$\lambda$ & $11.3(7.1,29.4)$ & $43.6(20.6,140.9)$ \\
$\sigma^{2}$ & $0.008(0.003,0.016)$ & $0.006(0.001,0.014)$ \\
\hline
\end{tabular}

Table 7: Parameter results (posterior medians and 95\% credible intervals) for two inefficiency distributions with $t$ errors for the electricity data with economic restrictions

where the function step (a) equals one if a is nonnegative and zero otherwise, and $\backslash$ indicates that the following line is the continuation of the current line and both should be entered as a single line.

It should be noted that the non-standard likelihood function can lead to a severe deterioration in the performance of the Gibbs sampler that WinBUGS implements, which can lead to slow convergence and mixing. In this example, we used a thinning of 100 which resulted in good autocorrelation properties in the chain. Table 7 presents results for two possible choices of inefficiency distribution. Comparison with Table 5 shows that the economic constraints are rarely violated for these data and the implementation of economic constraints in this example has little effect on the analysis. Only the inference on $\beta_{3}$ is moderately affected, as could be expected from the unrestricted output in Table 5.

\section{A Panel of US Hospital data}

Our second example reanalyses data on costs of US hospitals initially conducted in Koop, Osiewalski and Steel (1997), and we refer to the latter paper for further details and background of hospital cost estimation, the data and the particular frontier used. The data correspond to $N=382$ nonteaching U.S. hospitals over the years 1987-1991 ( $T=5)$, selected so as to constitute a relatively homogeneous sample. The frontier describing cost involves five different outputs $Y_{1}, \ldots, Y_{5}$ : number of cases, number of inpatient days, number of beds, number of outpatient visits and a case mix index. We also include a measure of capital stock, $C$, an aggregate wage index, $P$, and a time trend $t$ to capture any missing dynamics. We choose a flexible translog specification and impose linear homogeneity in prices, which allows us to normalize with respect to the price of materials. Thus, in the notation of (1) and dropping 
observational subscripts for ease of notation, $y=\log (\operatorname{cost})$ and $x^{\prime} \beta$ becomes:

$$
\begin{aligned}
x^{\prime} \beta= & \sum_{i=1}^{5} \beta_{i} \log Y_{i}+\beta_{6} \log P+\beta_{7}(\log P)^{2}+\sum_{i=1}^{5} \beta_{7+i} \log Y_{i} \log P+\beta_{13} \log C \\
& +\sum_{i=1}^{5} \beta_{13+i} \log Y_{i} \log C+\beta_{19} \log P \log C+\beta_{20}(\log C)^{2}+\beta_{21} t+\beta_{22} t^{2} \\
& +\sum_{i=1}^{5} \sum_{j=i}^{5} \beta_{22+5(i-1)+j} \log Y_{i} \log Y_{j} .
\end{aligned}
$$

In this section, we will use the normal sampling in (1) combined with an exponential inefficiency distribution, except for Subsection 4.3, where we assume truncated normal inefficiencies.

\subsection{Including covariates in the inefficiency distribution}

Koop, Osiewalski and Steel (1997) consider a method for extending the stochastic frontier to allow exponentially distributed inefficiencies to depend upon covariates. Their model assumes that each firm has a vector of binary covariates, $w_{i}$ for the $i$-th firm. That firm's inefficiency is modelled as

$$
u_{i} \sim \operatorname{Exp}\left(\exp \left\{w_{i}^{\prime} \gamma\right\}\right) .
$$

where $\gamma$ is a vector of coefficients. In the current example, there are 3 possible ownership categories for each firm and $w$ contains dummy variables to represent category membership. Actually, since WinBUGS does not rely on known forms for the conditionals, the covariates $w_{i}$ can also include non-binary variables. It seems reasonable to assume a priori that our belief about the efficiency distribution for each category should be the same, i.e.

$$
\exp \left\{\gamma_{j}\right\} \sim \operatorname{Exp}\left(-\log r^{\star}\right) .
$$

This model can be coded by defining lambda [i ], the inverse of mean inefficiency for the $i$-th firm and data 2 a matrix containing each firm's characteristics $w$.

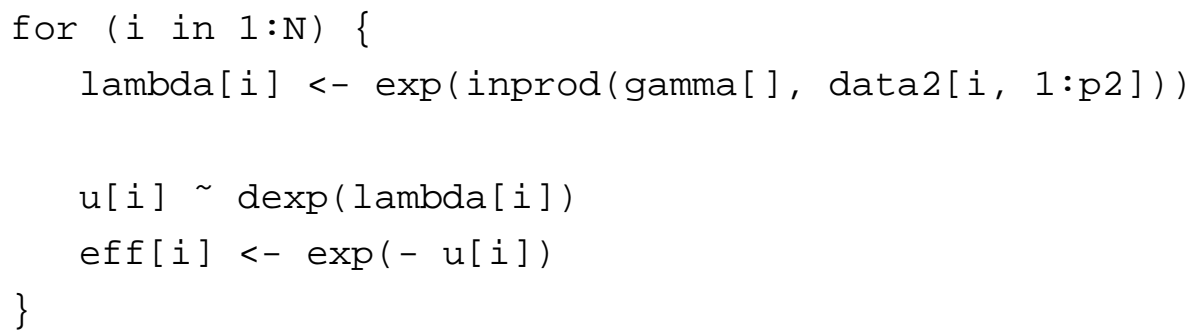

It is easier to define a prior distribution for $\exp \left\{\gamma_{j}\right\}$ and WinBUGS allows us to define the relationship gamma $[j]$ < $<-$ log (expgamma [j] ) using a logical node. The WinBUGS code for this prior is:

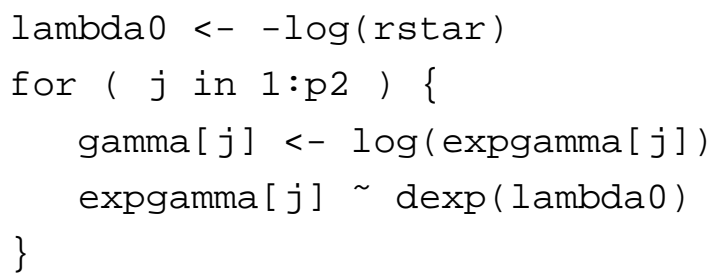


Using ownership dummies (indicating non-profit, for-profit or government-run hospitals corresponding to coefficients $\gamma_{1}, \gamma_{2}$ and $\gamma_{3}$, respectively) as covariates for the inefficiency distribution, Table 9 compares the DIC values for the basic model with that of the model including these covariates. There seems to be little support for this model extension. Nevertheless, Table 8 does indicate some evidence for lower efficiencies in the for-profit sector. The latter is in line with the results in Koop, Osiewalski and Steel (1997).

\begin{tabular}{|c|c|c|c|}
\hline & basic model & covariates & parametric time-varying eff. \\
\hline$\lambda$ & $5.75(5.07,6.51)$ & & $6.00(5.22,6.87)$ \\
$\exp \left\{\gamma_{1}\right\}$ & & $6.25(5.38,7.29)$ & \\
$\exp \left\{\gamma_{2}\right\}$ & & $4.04(3.12,5.16)$ & \\
$\exp \left\{\gamma_{3}\right\}$ & & $6.89(5.11,9.16)$ & \\
$\eta$ & & & $-0.026(-0.047,-0.0041)$ \\
$\sigma^{2}$ & $0.0042(0.0039,0.0045)$ & $0.0042(0.0039,0.0045)$ & $0.0042(0.0039,0.0045)$ \\
\hline
\end{tabular}

Table 8: Selected parameter results (posterior medians and $95 \%$ credible intervals) for the hospital data. $\gamma_{1}$ is the coefficient of the non-profit ownership dummy, while $\gamma_{2}$ and $\gamma_{3}$ correspond to, respectively, for-profit and governmentrun hospitals. Inefficiency distributions are exponential

\begin{tabular}{|l|c|c|c|c|}
\hline & $\bar{D}$ & $\hat{D}$ & $p_{D}$ & $D I C$ \\
\hline basic model & -5033 & -5413 & 380 & -4654 \\
exogenous variables & -5025 & -5403 & 378 & -4647 \\
par. time-varying & -5041 & -5423 & 382 & -4660 \\
\hline
\end{tabular}

Table 9: DIC results for the hospital data with various model specifications and exponential inefficiencies for the first three and truncated normal for the last two models

\subsection{Time-varying efficiency}

The assumption made in equation (2) that firm-specific technical efficiency is constant over time may not always be tenable. An alternative model that allows time-varying efficiencies uses $u_{i t}$ to represent the inefficiency of firm $i$ at time $t$. A simplifying assumption proposed in Lee and Schmidt (1993) defines $u_{i t}=\beta(t) u_{i}$. This specification is parsimonious but makes a strong assumption about the form of timedependence. Several functions have been considered for $\beta(t)$, in particular Battese and Coelli (1992) propose

$$
\beta(t)=\exp \{\eta(t-T)\}
$$

where positive $\eta$ indicates increasing efficiency over time. We shall denote this case as that of "parametric time-varying" efficiencies. We choose the prior distribution of $\eta$ to be a zero-mean normal distribution with variance 0.25 which represents our prior indifference between increasing and decreasing efficiency 
and, for $T=5$, supports reasonable predictive distributions of efficiency at each time point. This new model can be implemented in WinBUGS by changing the mean of the log costs to

$\mathrm{t}<-$ data $[\mathrm{k}, \mathrm{p}+2]$

$\mathrm{mu}[\mathrm{k}]<-$ alpha $+\exp (\operatorname{eta}(\mathrm{t}-\mathrm{T})) \star \mathrm{u}[\mathrm{firm}[\mathrm{k}]]+\operatorname{inprod}(\operatorname{beta}[], \operatorname{data}[\mathrm{k}, 1: \mathrm{p}])$

and the prior is represented by the statement

eta $\operatorname{dnorm}(0.0,4)$.

Table 9 indicates some support for this parametric time-varying model over the basic model. From the posterior results on $\eta$ in Table 8, we conclude that efficiencies tend to decrease somewhat over time.

\subsection{Truncated normal inefficiencies}

An alternative distribution for the inefficiencies is the truncated normal, as used in Stevenson (1980) and Battese and Coelli (1992). Battese and Coelli (1995) propose to use covariates to model the underlying mean of a truncated normal inefficiency distribution. Whereas they consider independent inefficiency terms, ${ }^{11}$ we will focus on the assumptions used earlier in this paper and first consider the case where inefficiencies are constant over time and distributed as

$$
u_{i} \sim \mathrm{N}^{+}\left(w_{i}^{\prime} \theta, \lambda^{-1}\right)
$$

where $w_{i}$ can group firm characteristics and $\theta$ is the associated $p_{3}$-dimensional parameter vector. The first element of $w_{i}$ is unity, so that the special case $p_{3}=1$ corresponds to i.i.d. truncated normal inefficiencies.

The implementation in WinBUGS is as follows:

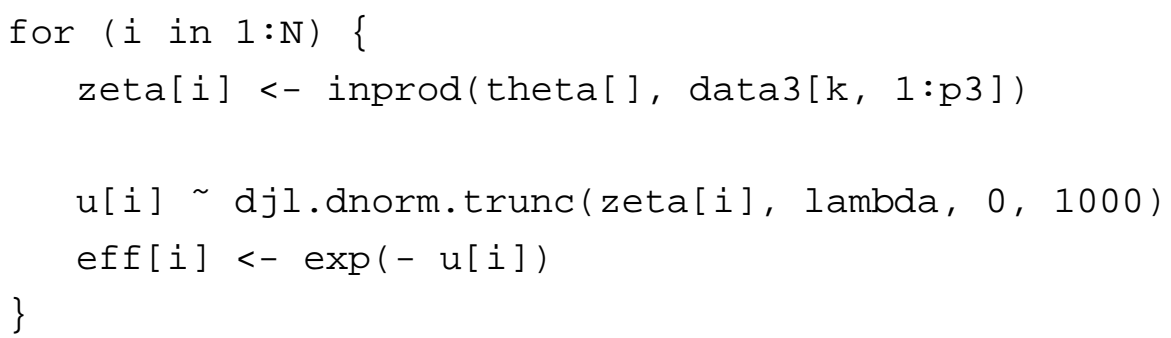

where data 3 groups the $w_{i}$ vectors into a $N \times p_{3}$ matrix.

The prior specification is based on Section 6 of van den Broeck et al. (1994), which covers the case when $p_{3}=1$ (no covariates) and proposes the use of a particular right-skewed skew-normal prior (see Azzalini, 1985) for the standardized underlying mean $\psi_{1}=\theta_{1} \sqrt{\lambda}$ and an independent gamma prior for $\lambda .^{12}$ In particular, we take

$$
p\left(\psi_{1}, \lambda\right)=2 \Phi\left(\psi_{1}\right) \phi\left(\psi_{1}\right) f_{G}\left(\lambda \mid 5,5 \log ^{2} r^{\star}\right),
$$

\footnotetext{
${ }^{11}$ Such a case is perfectly feasible within WinBUGS, and its implementation is described in the Appendix.

${ }^{12}$ As explained in van den Broeck et al. (1994), this leads to a half-Student distribution for $u_{i t}$ with 10 degrees of freedom and a prior median efficiency quite close to $r^{\star}$.
} 
where $\Phi(\cdot)$ and $\phi(\cdot)$ denote the cdf and pdf of a standard normal, and $f_{G}(\cdot \mid a, b)$ denotes the pdf of a $\mathrm{Ga}(a, b)$ distribution. In the more general case with covariates $\left(p_{3}>1\right)$ we extend this specification by adopting vague priors centred over zero for the other elements of the vector $\psi=\theta \sqrt{\lambda}$ :

$$
\psi_{i} \sim \mathrm{N}(0,10), i=2, \ldots, p_{3}
$$

after normalising any continuous covariates to have zero mean and unitary standard deviation.

Table 10 displays some results for the basic model $\left(p_{3}=1\right)$, and the model where we include the three ownership dummies as covariates in the inefficiency distribution. Again, we find that for-profit hospitals tend to be somewhat less efficient. The table also contains results for the model with timevarying efficiency, defined as in the previous subsection. Again, there is some evidence that efficiencies tend to go down over time, although not as quickly as in the model with exponential inefficiencies.

\begin{tabular}{|c|c|c|c|}
\hline & basic model & covariates & parametric time-varying eff. \\
\hline$\lambda$ & $63.4(53.0,74.7)$ & $64.0(53.4,75.6)$ & $62.1(51.5,74.4)$ \\
$\theta_{1}$ & $0.36(0.29,0.46)$ & $0.13(-0.05,0.32)$ & $0.35(0.28,0.45)$ \\
$\theta_{2}$ & & $0.30(0.08,0.60)$ & \\
$\theta_{3}$ & & $0.37(0.14,0.67)$ & \\
$\theta_{4}$ & & $0.28(0.05,0.58)$ & \\
$\eta$ & & & $-0.0018(-0.0036,0.0000)$ \\
$\sigma^{2}$ & $0.0038(0.0035,0.0041)$ & $0.0038(0.0036,0.0041)$ & $0.0038(0.0035,0.0041)$ \\
\hline
\end{tabular}

Table 10: Selected parameter results (posterior medians and 95\% credible intervals) for the truncated normal inefficiency distribution using the hospital data. For the model with covariates in the inefficiency distribution, $\theta_{1}$ is the intercept, $\theta_{2}$ the coefficient of the non-profit ownership dummy, while $\theta_{3}$ and $\theta_{4}$ correspond to, respectively, for-profit and government-run hospitals.

The DIC results for the truncated normal models, reported in Table 11, indicate a strong support for the truncated normal inefficiencies over the exponential ones in Subsections 4.1 and 4.2. There is no real support for the inclusion of covariates in the inefficiency distribution, and, as before, the parametric time-varying efficiency model seems the most preferred. In fact, the predictive efficiency distributions generated by the truncated normal models will have relatively little mass close to full efficiency, and a mode around 0.7 or so, very much in line with the results obtained on the same data with a nonparametric inefficiency distribution in Griffin and Steel (2004a) and with a flexible parametric inefficiency distribution in Griffin and Steel (2004b). This is in contrast with the exponential and half-normal models, which are constrained to put a mode at full efficiency (see Figure 4).

However, due to the more flexible nature of the inefficiency distribution, the models with truncated normal inefficiency distributions take considerably longer to run in WinBUGS, as the sampler does not mix very well and, thus, we need to thin the chain considerably. A more efficient implementation would require a tailored MCMC scheme that takes into account the difficulty of distinguishing between the 


\begin{tabular}{|l|c|c|c|c|}
\hline & $\bar{D}$ & $\hat{D}$ & $p_{D}$ & $D I C$ \\
\hline basic model & -5215 & -5611 & 395 & -4820 \\
exogenous variables & -5214 & -5611 & 396 & -4818 \\
par. time-varying & -5232 & -5630 & 398 & -4834 \\
\hline
\end{tabular}

Table 11: DIC results for the hospital data with various model specifications and truncated normal inefficiencies.

intercept and the inefficiency mean. Thus, we can only recommend the standard WinBUGS code ${ }^{13}$ provided here with the warning that long chains may be required as convergence can be fairly slow for truncated normal models. The same type of problem may also affect other flexible inefficiency distributions, such as the gamma.

\subsection{Model comparison of the form of the frontier}

The stochastic frontier represents an approximation to the best-practice output for a set of inputs or lowest cost for producing a set of outputs. The common forms of the function have usually been loglinear but there may be uncertainty in the frontier specification. For example, a Cobb-Douglas functional form is fairly restricted. A translog is much more flexible, using a second order approximation to the unknown function, but at the expense of introducing many parameters which can lead to a poor fit. An improvement in fit may arise through careful selection of the higher order terms and interactions or of various other covariates. In the example above, we might wish to allow for the possibility of no time trend, a linear time trend or a quadratic time trend. In the Bayesian approach these questions of model choice can be answered through model comparison tools such as the DIC. We restrict attention to deciding on the type of time trend to include in the model. Table 12 shows the results of running three separate chains incorporating no time trend, a linear trend and a quadratic time trend in the basic model with a common exponential inefficiency distribution for all hospital types and constant efficiencies over time. The models with a time trend far outperform the model without time trend. The results indicate a preference for the model with a quadratic time effect, as used in the previous subsections.

\section{Conclusions}

This paper demonstrates that WinBUGS provides a useful framework for the Bayesian analysis of stochastic frontier models. We provide code to implement a standard model for cross-sectional, balanced and unbalanced panel data with a time-invariant exponential inefficiency distribution. Many other,

\footnotetext{
${ }^{13}$ This could be much improved by a specialized add-on to WinBUGS for stochastic frontier models with flexible inefficiency distributions. In particular, the parameterisation can be an important issue and we have found it to be a key factor in the mixing properties of the MCMC chain in problems where the inefficiency distribution is hard to distinguish from the measurement error distribution, such as general gamma distributions (Griffin and Steel, 2004b) or even nonparametric distributions (Griffin and Steel, 2004a). In both papers, we used the device of centring, which is explained in this context in Griffin and Steel (2004a). However, the implementation of centring is not trivial in WinBUGS and would require writing a specific add-on.
} 


\begin{tabular}{|l|c|c|c|c|}
\hline & $\bar{D}$ & $\hat{D}$ & $p_{D}$ & $D I C$ \\
\hline quadratic time trend & -5033 & -5413 & 380 & -4654 \\
linear time trend & -5026 & -5405 & 379 & -4647 \\
no time trend & -2581 & -2914 & 333 & -2248 \\
\hline
\end{tabular}

Table 12: DIC results for the hospital data with the basic model and exponential inefficiencies

more complicated, models can be analysed using simple extensions of this code, for example to include covariates in the efficiency distribution and time-varying efficiencies. We also illustrate ways to impose economic regularity conditions and deal with model uncertainty. We emphasize that we have merely shown a few possible model specifications and extensions ${ }^{14}$ and it should also be fairly straightforward to implement many other Bayesian models such as random coefficient frontiers (see Tsionas, 2002 and Huang, 2004) or models developed in the literature for dealing with multiple-output analysis or the modelling of undesirable outputs (see Fernández, Koop and Steel, 2002). We also stress that priors can be specified in line with genuine prior beliefs, as WinBUGS does not require priors to be conjugate in any sense. Modelling, both of the sampling model and the prior assumptions, can be conducted creatively and in accordance with the particular problem at hand, without worrying about having to develop and modify complicated computer code.

WinBUGS immediately leads to full posterior distributions of the model parameters and interesting functions of these parameters, such as firm-specific efficiencies and the rankings of firm-specific efficiencies. Many graphical and other summaries of the posterior distributions and the behaviour of the MCMC sampler are built-in. The availability of CODA-compatible output allows a range of convergence diagnostics to be produced very easily. WinBUGS can be called from within other programs and interfaces with a variety of other popular environments (R, Stata, SAS, Matlab) are possible, as explained on the WinBUGS website. In order to improve the efficiency of inference under regularity conditions or using the more flexible inefficiency distributions, an add-on to WinBUGS specifically written to take advantage of the structure of the problem should constitute a big improvement. However, the user-friendly and generic structure of WinBUGS could, in our view, make a substantial contribution to opening up the area of stochastic frontiers to applied practitioners. In particular, it allows researchers to conduct Bayesian inference with these models with a relatively small initial investment and stimulates easy experimentation with the structure of the basic model.

We would certainly recommend the applied user of stochastic frontier models to experiment with WinBUGS and we hope that the availability of WinBUGS code (freely available on the web) will allow these users to add Bayesian methods to their modelling and inference toolbox.

\section{Appendix: Implementing the Battese and Coelli (1995) model}

A model with more flexible time dependence of the efficiencies (and less panel structure), which

\footnotetext{
${ }^{14}$ Although our paper covers perhaps the most popular models for typical applications.
} 
also incorporates covariate information in the efficiency distribution is that of Battese and Coelli (1995). In particular, Battese and Coelli (1995) propose the use of covariates, possibly depending on firms and time, to model the underlying mean of a truncated Normal inefficiency distribution. In WinBUGS this is easily implemented by changing the specification of mu to

$m u[k]<-a l p h a+u[k]+i n p r o d(b e t a[], \operatorname{data}[k, 1: p])$

while the inefficiency term corresponds to $u_{i t} \sim \mathrm{N}^{+}\left(w_{i t}^{\prime} \theta, \lambda^{-1}\right)$, where $w_{i t}$ can group firm characteristics, which may be time dependent, and time-related variables, and inefficiency is now modelled as

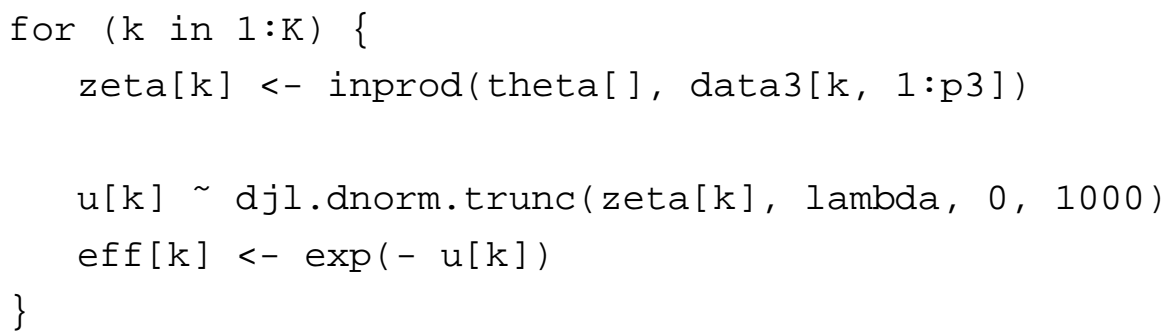

where data 3 groups the $w_{i t}$ vectors into a $K \times p_{3}$ matrix.

\section{References}

Aigner, D., C.A.K. Lovell and P. Schmidt. (1977). "Formulation and estimation of stochastic frontier production function models." Journal of Econometrics 6, 21-37.

Arickx, F., J. Broeckhove, M. Dejonghe and J. van den Broeck. (1997). "BSFM: A Computer Program for Bayesian Stochastic Frontier Models." Computational Statistics 12, 403-421.

Azzalini, A. (1985). "A Class of Distributions which Includes the Normal Ones.” Scandinavian Journal of Statistics 12, 171-178.

Battese, G.E. and T.J. Coelli. (1992). "Frontier Production Functions, Technical Efficiency and Panel Data: With Application to Paddy Farmers in India." Journal of Productivity Analysis 3, 153-169.

Battese, G.E. and T.J. Coelli. (1995). "A Model for Technical Inefficiency Effects in a Stochastic Frontier Production Function for Panel Data." Empirical Economics 20, 325-332.

Christensen, L. R. and W. H. Greene (1976). "Economies of Scale in U.S. Electric Power Generation." Journal of Political Economy 84, 655-676.

Dorfman, J.H. and G. Koop (2005). "Current Developments in Productivity and Efficiency Measurement." Special issue of Journal of Econometrics, 126, 233-570.

Ennsfellner, K.C., D. Lewis D and R.I. Anderson. (2004). " Production efficiency in the Austrian insurance industry: A Bayesian examination.” Journal of Risk and Insurance 71, 135-159. 
Fernández, C., G. Koop and M. F. J. Steel. (2002). "Multiple Output Production with Undesirable Outputs: An Application to Nitrogen Surplus in Agriculture," Journal of the American Statistical Association, 97, 432-442.

Greene, W.H. (1990). “A Gamma-Distributed Stochastic Frontier Model.” Journal of Econometrics 46, 141-163.

Griffin, J. E. and Steel, M. F. J. (2004a). "Semiparametric Bayesian Inference for Stochastic Frontier Models.” Journal of Econometrics 123, 121-152.

Griffin, J. E. and Steel, M. F. J. (2004b). "Flexible Mixture Modelling of Stochastic Frontiers." University of Warwick, Technical Report.

Huang, H.C. (2004). "Estimation of technical inefficiencies with heterogeneous technologies." Journal of Productivity Analysis 21, 277-296.

Kim, Y. and P. Schmidt (2000). "A review and empirical comparison of Bayesian and classical approaches to inference on efficiency levels in stochastic frontier models with panel data." Journal of Productivity Analysis, 14, 91-118.

Koop, G. (1999). "Bayesian Analysis, Computation and Communication.” Journal of Applied Econometrics $14,677-689$.

Koop, G., J. Osiewalski and M.F.J. Steel (1997). "Bayesian Efficiency Analysis Through Individual Effects: Hospital Cost Frontier.” Journal of Econometrics, 76, 77-105.

Koop, G, M.F.J. Steel and J. Osiewalski. (1995). "Posterior analysis of stochastic frontier models using Gibbs sampling." Computational Statistics, 10, 353-373.

Kumbhakar, S.C. and Lovell, C.A.K. (2000). Stochastic Frontier Analysis. Cambridge University Press: New York.

Kumbhakar, S.C. and E.G. Tsionas. (2005). "Measuring technical and allocative inefficiency in the translog cost system: a Bayesian approach.” Journal of Econometrics, 126, 355-384.

Kurkalova L.A. and A. Carriquiry. (2002). "An analysis of grain production decline during the early transition in Ukraine: A Bayesian inference." American Journal of Agricultural Economics 84, 1256-1263.

Lee, Y. H. and P. Schmidt (1993). "A production frontier model with flexible temporal variation in technical efficiency." in H. O. Fried, C. A. K. Lovell, and S. S. Schmidt, eds. The Measurement of Productive Efficiency: Techniques and Applications. New York: Oxford University Press.

Meeusen, W. and J. van den Broeck. (1977). "Efficiency estimation from Cobb-Douglas production functions with composed errors." International Economic Review, 8, 435-444.

Spiegelhalter D. J., N. G. Best, B. P. Carlin and A. van der Linde (2002). "Bayesian measures of model complexity and fit (with discussion)." Journal of the Royal Statistical Society B, 64, 583-640.

Stevenson, R.E. (1980). “Likelihood functions for generalized stochastic frontier estimation.” Journal of Econometrics 13, 57-66. 
Terrell, D. (1996). "Incorporating monotonicity and concavity conditions in flexible functional forms." Journal of Applied Econometrics 11, 179-194.

Tsionas, E. G. (2000). "Full likelihood inference in Normal-gamma stochastic frontier models." Journal of Productivity Analysis, 13, 183-205.

Tsionas, E. G. (2002). "Stochastic Frontier Models with Random Coefficients." Journal of Applied Econometrics, 17, 127-147.

van den Broeck, J., G. Koop, J. Osiewalski and M.F.J. Steel. (1994). "Stochastic Frontier Models: A Bayesian Perspective." Journal of Econometrics, 61, 273-303. 